\title{
The influence of depth of anesthesia on motor evoked potential response during awake craniotomy
}

\author{
Shunya Ohtaki, MD, ${ }^{1}$ Yukinori Akiyama, MD, PhD, ${ }^{1}$ Aya Kanno, MD, ${ }^{1}$ Shouhei Noshiro, MD,1 \\ Tomo Hayase, MD, PhD, ${ }^{2}$ Michiaki Yamakage, MD, PhD, ${ }^{2}$ and Nobuhiro Mikuni, MD, PhD' \\ Departments of ${ }^{1}$ Neurosurgery and ${ }^{2}$ Anesthesiology, Sapporo Medical University School of Medicine, Sapporo, Hokkaido, Japan
}

OBJECTIVE Motor evoked potentials (MEPs) are a critical indicator for monitoring motor function during neurological surgery. In this study, the influence of depth of anesthesia on MEP response was assessed.

METHODS Twenty-eight patients with brain tumors who underwent awake craniotomy were included in this study. From a state of deep anesthesia until the awake state, MEP amplitude and latency were measured using 5 -train electrical bipolar stimulations on the same site of the precentral gyrus each minute during the surgery. The depth of anesthesia was evaluated using the bispectral index (BIS). BIS levels were classified into 7 stages: $<40$, and from 40 to 100 in groups of 10 each. MEP amplitude and latency of each stage were compared. The deviation of the MEP measurements, which was defined as a fluctuation from the average in every BIS stage, was also considered.

RESULTS A total of 865 MEP waves in 28 cases were evaluated in this study. MEP amplitude was increased and latency was decreased in accordance with the increases in BIS level. The average MEP amplitudes in the > $90 \mathrm{BIS}$ level was approximately 10 times higher than those in the $<40$ BIS level. Furthermore, the average MEP latencies in the $>90$ BIS level were 1.5-3.1 msec shorter than those in the $<60$ BIS level. The deviation of measured MEP amplitudes in the $>90 \mathrm{BIS}$ level was significantly stabilized in comparison with that in the $<60 \mathrm{BIS}$ level.

CONCLUSIONS MEP amplitude and latency were closely correlated with depth of anesthesia. In addition, the deviation in MEP amplitude was also correlated with depth of anesthesia, which was smaller during awake surgery (high BIS level) than during deep anesthesia. Therefore, MEP measurement would be more reliable in the awake state than under deep anesthesia.

http://thejns.org/doi/abs/10.3171/2015.11.JNS151291

KEY WORDS anesthesia depth; motor evoked potential; bispectral index; diagnostic and operative techniques

I NTRAOPERATIVE neurophysiological monitoring of motor evoked potentials (MEPs) has been widely used to evaluate motor function during neurological surgeries., ${ }^{3,11}$ There are 2 different methods for MEP detection: a muscle MEP to be recorded from the muscles and a spinal MEP to be recorded over the dura of the spinal cord. Although muscle MEP recording is more commonly used than spinal MEP recording because it is less invasive and superior at the point of identifying the functional localization on the cerebral cortex, it is affected by multiple factors such as body temperature, stimulation conditions, anesthetic agents, and depth. It has been reported that muscle MEPs are suppressed by midazolam, volatile anesthetics, and even propofol in a dose-dependent manner. ${ }^{4,9,10,12,14,16,20,21,25,28,29}$ Since muscle MEPs are known to be sensitive to volatile anesthetics, intravenous anesthetics are preferentially used during muscle MEP monitoring..$^{12,25}$

The bispectral index (BIS) is one of several methods used to monitor the depth of anesthesia. Since the BIS was introduced by Aspect Medical Systems, Inc. in 1994, it has been studied widely and has routinely been used to monitor the depth of anesthesia., $717,26,27$

To clarify the influence of depth of anesthesia on muscle MEPs, we have studied the relationship between BIS

ABBREVIATIONS BIS = bispectral index; EEG = electroencephalography; EMG = electromyography; $G A B A=\gamma$-aminobutyric acid; MEP = motor evoked potential. SUBMITTED June 5, 2015. ACCEPTED November 3, 2015.

INCLUDE WHEN CITING Published online March 4, 2016; DOI: 10.3171/2015.11.JNS151291. 
and muscle MEP responses, such as amplitude, latency, and deviation, by using continuous cortical electric stimulation during awake craniotomy.

\section{Methods}

\section{Patients}

This study was approved by the Sapporo Medical University Institutional Review Board. Informed consent was obtained preoperatively from all patients. Included in this study were 28 patients ranging in age from 24 to 80 years (median 57 years) who had a brain tumor located close to the pyramidal tract and underwent awake surgery (Table 1).

\section{BIS Monitoring}

The BIS was recorded before the induction of anesthesia and throughout the operative procedure (BIS, Philips). The BIS was automatically calculated and displayed every 5 seconds, which represented the electroencephalography (EEG) activity during the previous 60 seconds. The BIS was reported as a unitless whole number between 0 and 100 . The 1-piece design of the patch electrode was positioned from the contra- and ipsilateral nasal bones to the ipsilateral zygomatic bone to avoid any interference due to craniotomy.

\section{Anesthesia}

Routine physiological monitoring including electrocardiography, $\mathrm{PO}_{2}$ and $\mathrm{PCO}_{2}$ monitoring, and temperature was started and then recorded while the patient breathed $100 \%$ oxygen. General anesthesia was induced with a single dose of propofol (2 mg/kg) and fentanyl (1-2 $\mu \mathrm{g} / \mathrm{kg})$. To facilitate laryngeal mask airway insertion, vecuronium bromide $(0.1 \mathrm{mg} / \mathrm{kg})$ was injected immediately after the loss of consciousness. Muscle relaxants were administered only for intubation and were not continued during surgical procedures. A peripheral nerve stimulator was used

TABLE 1. Summary of the clinical characteristics of 28 patients with brain tumors

\begin{tabular}{cc}
\hline Tumor Type \& Location & No. of Patients \\
\hline Histological type & \\
\hline Pleomorphic xanthoastrocytoma & 1 \\
\hline Diffuse astrocytoma & 4 \\
\hline Oligoastrocytoma & 1 \\
\hline Oligodendroglioma & 4 \\
\hline Anaplastic astrocytoma & 2 \\
\hline Anaplastic oligodendroglioma & 4 \\
\hline Glioblastoma & 1 \\
\hline Gliosarcoma & 2 \\
\hline Others & \\
\hline Anatomical location (right/left) & $7 / 5$ \\
\hline Frontal lobe & $5 / 2$ \\
\hline Temporal lobe & $2 / 1$ \\
\hline Insular cortex & $3 / 3$ \\
\hline Parietal lobe & \\
\hline
\end{tabular}

to confirm a train-of-four muscle contraction by complete reversal of muscle relaxant effect using TOF-Watch (Mammendorfer Institut für Physik und Medizin GmbH). During the first stage of the operation, general anesthesia was maintained with oxygen (33\%) and propofol infusion using a BIS within the range of $40 \pm 5$. Ventilation was controlled to maintain end-tidal $\mathrm{PaCO}_{2}$ at approximately $35-40 \mathrm{~mm} \mathrm{Hg}$. Body temperature was maintained within $36^{\circ}-37^{\circ} \mathrm{C}$ using a warming blanket. Extubation was performed at 65-90 (median 72) BIS levels during operative procedures in all patients.

\section{MEP Measurement}

The bilateral abductor pollicis brevis and tibialis anterior muscles were examined using the electromyography (EMG) recording. ${ }^{22}$ MEPs were recorded using a Digitimer MultiPulse Stimulator (Neuromaster MEE-1232, Nihon Kohden) with electrodes placed on the surface of the precentral gyrus, which was determined using an intraoperative navigation system and confirmed by the somatosensory evoked potential reversal method..$^{22}$ Cortical stimulation consisted of a train-of-five 200 - $\mu$ sec-duration pulses with a 2-msec interval. The amplitude of the MEPs was evaluated by measuring peak-to-peak differences, whereas latency was defined as the span between the start of the stimulation in a given sequence and the first assessable amplitude. The deviations in the MEP measurements were also evaluated. MEPs were recorded every minute after general anesthesia until the awake state during awake craniotomy.

\section{Data Acquisition, Analyses, and Statistics}

The MEPs recorded during the operative procedure near the pyramidal tract were excluded to eliminate the effects of surgical maneuver for MEP recordings.

The BIS levels were classified into 7 stages, with $<40$ as BIS level stage 1, and from 40 to 100 (in groups of 10) divided into 6 more stages. The MEP amplitudes and latencies were compared among these 7 BIS stages. In addition, the deviation in the MEP measurements, which was defined as a fluctuation from the average in every BIS stage, was also evaluated.

Data are expressed as mean \pm SD or SE. Statistical analysis was performed using IBM SPSS 22.0 (IBM Inc.). Statistical difference was assessed using Spearman's rank correlation coefficient and 1-way ANOVA followed by the Tukey test, and $p<0.05$ was considered statistically significant.

\section{Results}

Monitoring of MEP responses was recorded from deep anesthesia through the awake state in all patients. Of a total 865 MEP responses, the number of MEP responses were $56,160,145,119,94,155$, and 136 waves at $0-39,40-$ 49, 50-59, 60-69, 70-79, 80-89, and 90-100 BIS levels, respectively. Representative MEP responses at each BIS level, which were obtained from a 67-year-old woman, are shown in Fig. 1.

The mean peak-to-peak amplitudes $( \pm \mathrm{SE})$ of all the MEPs were $194.9 \pm 30.2 \mu \mathrm{V}$ at the $0-39$ BIS level, 339.1 

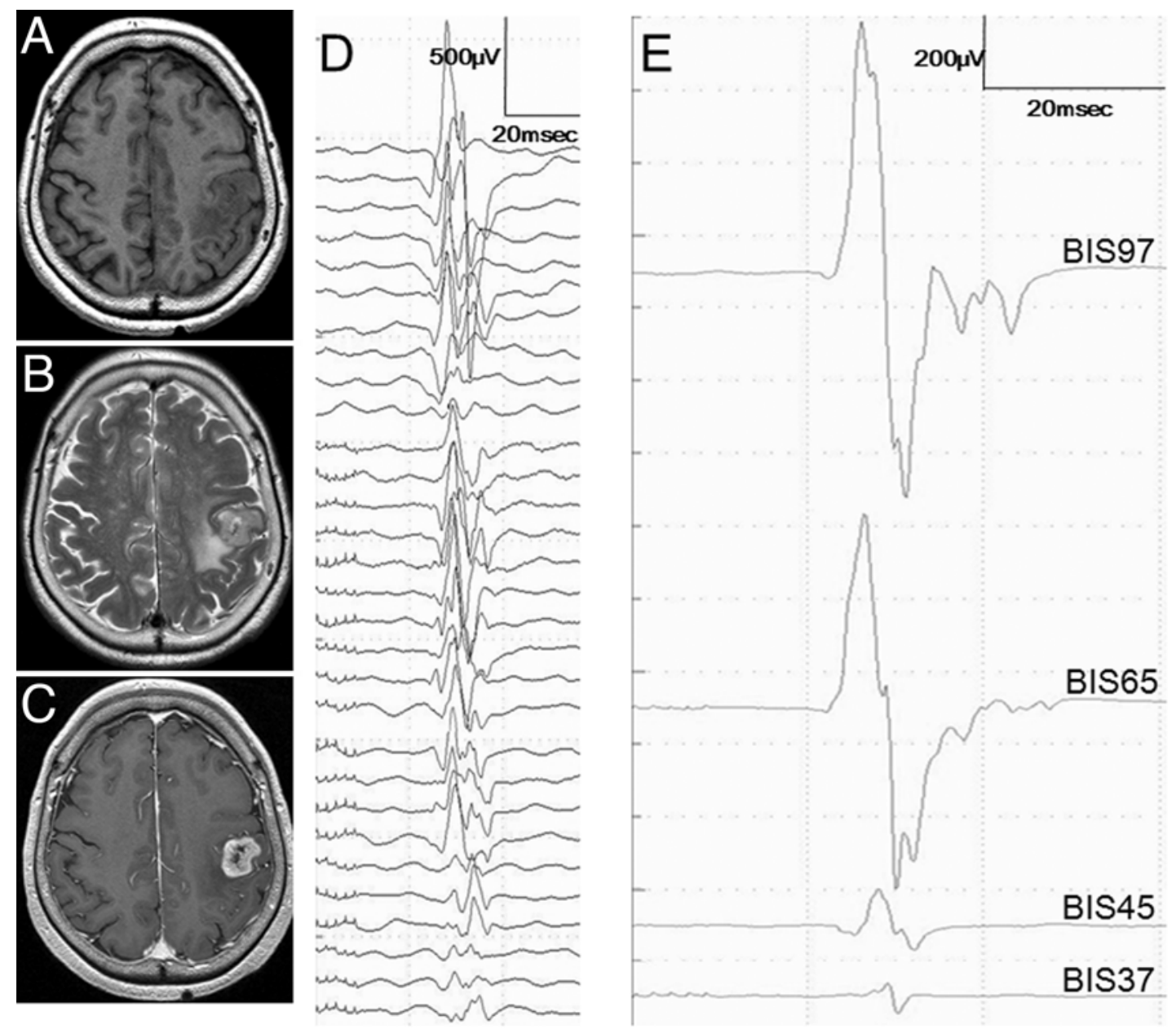

FIG. 1. Intraoperative MEP responses obtained in a 67-year-old woman harboring a glioblastoma. A-C: A mass lesion located at the left parietal lobe is seen on axial T1-weighted (A), T2-weighted (B), and T1-weighted Gd-enhanced (C) MR images. D: MEP responses recorded every minute from deep anesthesia (lower) to the awake state (upper). E: MEP responses recorded by the same stimulus intensity at $37,45,65$, and 97 BIS levels.

$\pm 28.5 \mu \mathrm{V}$ at the $40-49 \mathrm{BIS}$ level, $231.2 \pm 21.3 \mu \mathrm{V}$ at the $50-59$ BIS level, $342.8 \pm 43.0 \mu \mathrm{V}$ at the 60-69 BIS level, $850.0 \pm 76.4 \mu \mathrm{V}$ at the $70-79 \mathrm{BIS}$ level, $1025.4 \pm 81.6 \mu \mathrm{V}$ at the $80-89 \mathrm{BIS}$ level, and $1314.0 \pm 67.8 \mu \mathrm{V}$ at the $90-100$ BIS level (Fig. 2 right). MEP responses at higher BIS levels exhibited comparatively larger amplitudes $(p<0.001)$.

The onset latencies of all MEPs $( \pm$ SE) at each BIS stage were as follows: $26.1 \pm 0.23,24.5 \pm 0.13,25.2 \pm 0.16$, $24.6 \pm 0.16,23.0 \pm 0.16,23.4 \pm 0.19$, and $22.9 \pm 0.14 \mathrm{msec}$, as shown in Fig. 3 right. MEP latencies in the $>90$ BIS levels were shorter than those in the $<70$ BIS levels ( $p<$ $0.001)$.

The MEP amplitudes were not stabilized at lower BIS levels as shown in Figs. 1D and 4 left. The averages of deviations $( \pm \mathrm{SD})$ in each BIS stage were $38.7 \% \pm 33.6 \%$, $37.7 \% \pm 30.4 \%, 30.3 \% \pm 27.2 \%, 26.8 \% \pm 21.4 \%$, and $23.1 \%$ $\pm 17.5 \%$ at the $0-59,60-69,70-79,80-89$, and $90-100$ BIS levels, respectively. In deep anesthesia, MEP amplitudes fluctuated between approximately $40 \%$ on the average and up to $80 \%$, but for the awake state, the deviation rates were halved from that of controls. The deviation in the MEP amplitudes was more stabilized at the $>90$ BIS level than at the $<60$ BIS levels (p < 0.01) (Fig. 4 right). On the other hand, deviation in the measured MEP latencies was not significantly different among each BIS level (data not shown).

\section{Discussion}

There has been no report so far showing a correlation between the anesthesia effects and MEP responses during deep anesthesia through the awake state. In the present study, it was demonstrated that MEP amplitude and latency were correlated closely with depth of anesthesia. MEP amplitude was increased and latency was decreased in accordance with increases in BIS levels. The average of the MEP amplitudes in the $>90$ BIS level was approximately 10 times higher than those in the $<40$ BIS levels. The average of MEP latencies were from 1.5 to $3.1 \mathrm{msec}$ shorter in the $>90$ BIS level than those in the $<60$ BIS levels. It is well known that MEPs are suppressed by most anesthesia agents, including propofol, which has a rapid onset of action and is quickly removed from the bloodstream by redistribution and metabolism in a dose-dependent manner. ${ }^{6,8}$ 


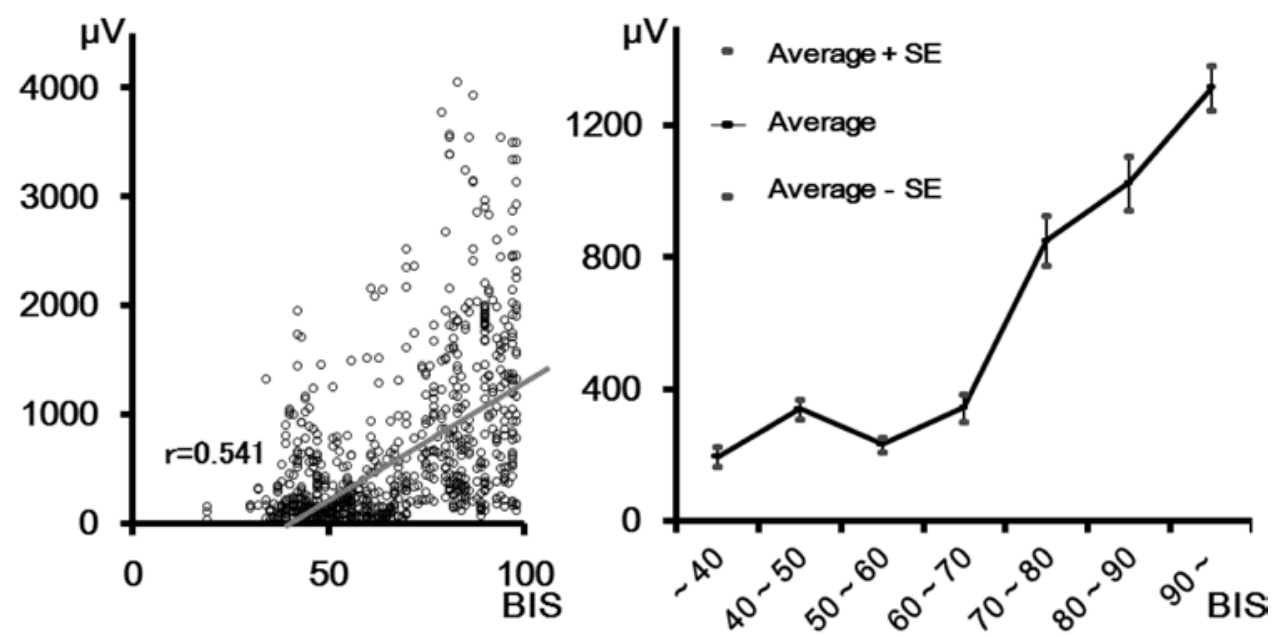

FIG. 2. Left: Scatter diagram showing a positive correlation between the peak-to-peak amplitude and BIS ( $p<0.001)$. Right: The mean peak-to-peak MEP amplitudes obtained in each BIS level. The mean MEP amplitude in BIS level $>90$ was higher than those in the other BIS levels $(p<0.01)$.

There are 3 kinds of $\gamma$-aminobutyric acid (GABA) receptors: $\mathrm{GABA}_{\mathrm{A}}, \mathrm{GABA}_{\mathrm{B}}$, and $\mathrm{GABA}_{\mathrm{C}}$. $\mathrm{GABA}_{\mathrm{A}}$ is mostly distributed in the central nervous system and is affected by anesthetics including propofol, which prolong the duration of GABAergic inhibitory postsynaptic currents. ${ }^{24}$ Propofol increases the threshold of $\alpha$-motor neurons in the spinal cord, which induce a reduction of the amplitude and a delay in the MEP latency. ${ }^{13,15}$ Although some authors have reported that an increasing propofol concentration reduced MEP amplitude in a dose-dependent manner with no effect on latency, $1,2,23$ our data demonstrated that deeper anesthesia not only induced a reduction of the MEP amplitude but also induced a delay in the MEP latency.

In our study, it was shown that the deviation in the measured MEP amplitudes was also correlated with BIS levels. While BIS levels were $<60$, which represents the state of deep anesthesia, the deviation in the measured MEP amplitudes was $40 \%$ to $80 \%$ from the averaged control.
Therefore, it would be difficult to distinguish whether the reduction in the MEP amplitude was due to a surgically injured pyramidal tract or a measurement deviation in deep anesthesia. It was reported that $>15 \%$ extension of the latency or $80 \%$ reduction of the MEP amplitude should be considered as a warning criterion for brain tumor resections. ${ }^{18}$ Krieg et al. reported that MEP monitoring was successful in 53 of 56 cases (93\%) and reduction of the MEP amplitude better correlated with postoperative outcomes when the threshold for the significant amplitude reduction was set at $80 \%$ during resection of metastases in motoreloquent brain regions. ${ }^{19}$ However, it is difficult to distinguish whether the reduction in MEP amplitudes was due to surgical manipulation of the pyramidal tract or anesthesia effects in deep anesthesia. On the other hand, while the BIS was $>90$, the deviation rate was $25 \%$ to $40 \%$, which is why awake monitoring could be more precise and easier to determine the mechanism of MEP reduction.
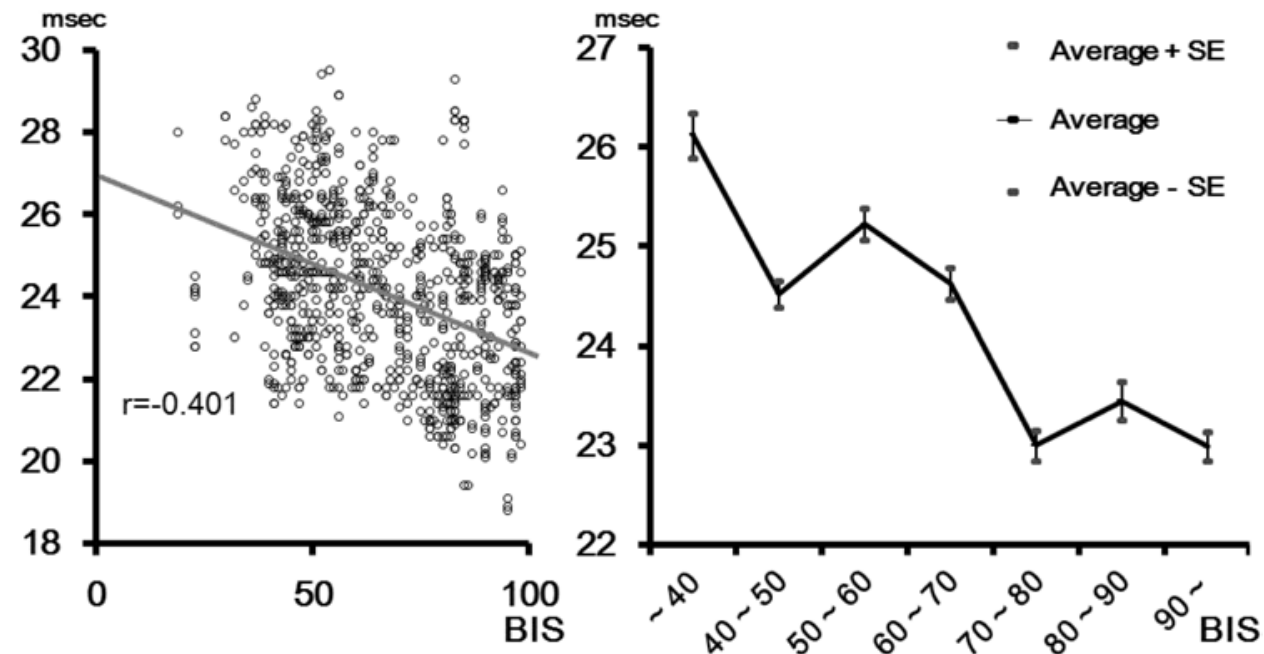

FIG. 3. Left: Scatter diagram showing a negative correlation between the MEP latency and BIS $(p<0.001)$. Right: The mean latencies of MEPs obtained in each BIS level. MEP latencies in the $>90$ BIS level were shorter than those in the $<70$ BIS levels $(p$ $<0.001$ ). 


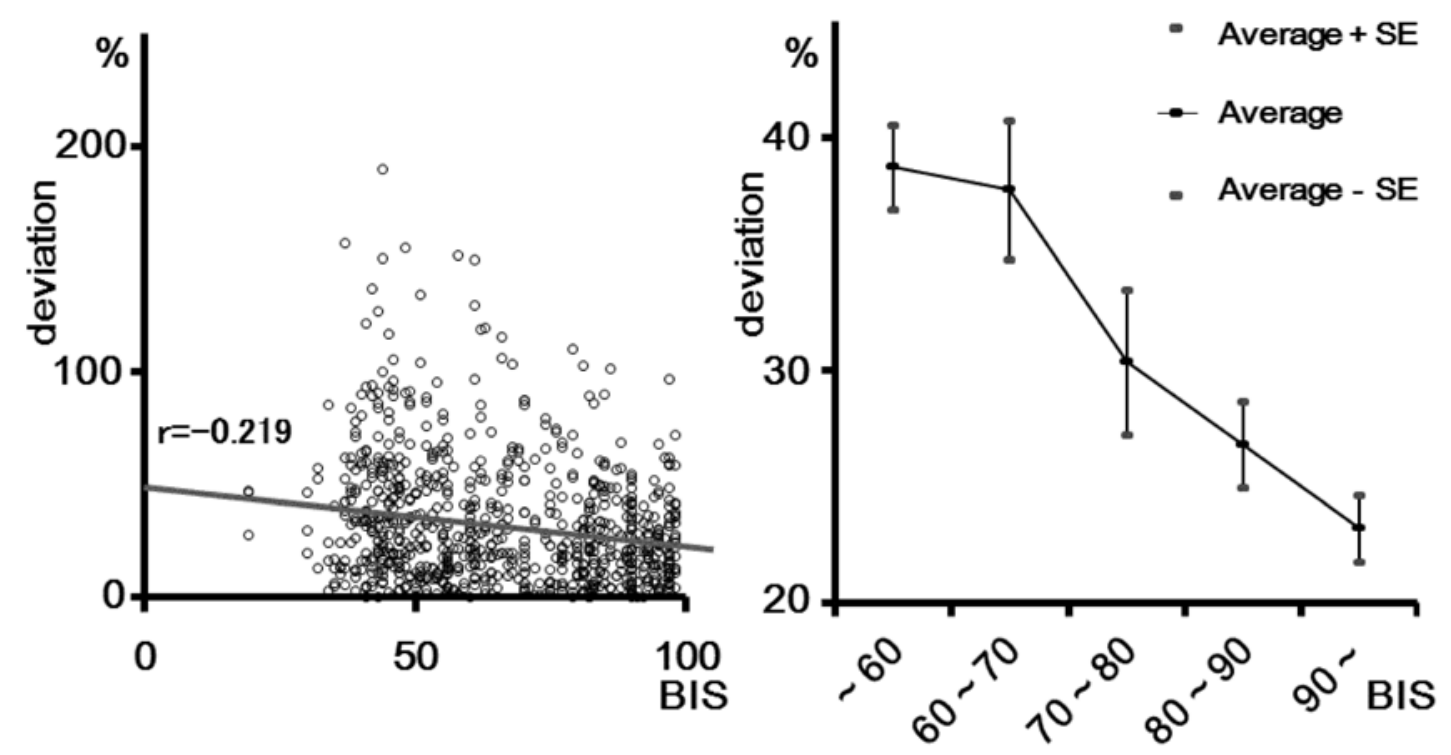

FIG. 4. Left: Scatter diagram showing a negative correlation between the deviation ratio of MEP amplitude and BIS ( $p<$ 0.001). Right: The stability of the amplitudes in each BIS level. The deviation in the MEP amplitudes was smaller in the $>90 \mathrm{BIS}$ level than that in the $<70$ BIS levels $(p<0.01)$.

There are several limitations to be considered in our study. The first is the reliability of BIS for anesthesia level. BIS analysis is based on 3 elements of the EEG: the burst suppression ratio, relative alpha/beta ratio, and bicoherence of the EEG. ${ }^{5}$ Aspect Medical Systems explained that EMG, which is recordable from the muscles of the head, is also included in the calculation when the BIS value is increased (Aspect Medical Systems, Inc.: Technology Overview: BIS 1997). There cannot be a one-to-one correspondence between EEG and BIS values since BIS values do not reflect real-time depth of anesthesia. The second limitation is that there is large individual variability. Individual differences in the MEP amplitudes and latencies vary widely. The third limitation is that it is difficult to evaluate the absolute MEP value, and hence, the study is limited to assessing the relative evaluation for MEP responses. In addition, it has to be taken into consideration that MEP recordings in the awake state could lead to intraoperative seizure and patient discomfort due to electrical stimulation.

\section{Conclusions}

We demonstrated in this study that the MEP amplitude changes with the depth of anesthesia monitored by the BIS. The average of the MEP amplitude in high BIS levels was stabilized, which was associated with increasing the signal-to-noise ratio. Further studies to evaluate the clinical usefulness of monitoring MEP responses associated with anesthetic level must be taken into consideration.

\section{References}

1. Bai D, Zhu G, Pennefather P, Jackson MF, MacDonald JF, Orser BA: Distinct functional and pharmacological properties of tonic and quantal inhibitory postsynaptic currents mediated by gamma-aminobutyric acid(A) receptors in hippocampal neurons. Mol Pharmacol 59:814-824, 2001
2. Bonin RP, Orser BA: GABA(A) receptor subtypes underlying general anesthesia. Pharmacol Biochem Behav 90:105112,2008

3. Calancie B, Harris W, Broton JG, Alexeeva N, Green BA: "Threshold-level" multipulse transcranial electrical stimulation of motor cortex for intraoperative monitoring of spinal motor tracts: description of method and comparison to somatosensory evoked potential monitoring. J Neurosurg 88:457-470, 1998

4. Calancie B, Klose KJ, Baier S, Green BA: Isoflurane-induced attenuation of motor evoked potentials caused by electrical motor cortex stimulation during surgery. J Neurosurg 74:897-904, 1991

5. Chan MT, Gin T: What does the bispectral EEG index monitor? Eur J Anaesthesiol 17:146-148, 2000

6. Cockshott ID: Propofol ('Diprivan') pharmacokinetics and metabolism-an overview. Postgrad Med J 61 (Suppl 3):45-50, 1985

7. Glass PS, Bloom M, Kearse L, Rosow C, Sebel P, Manberg $\mathrm{P}$ : Bispectral analysis measures sedation and memory effects of propofol, midazolam, isoflurane, and alfentanil in healthy volunteers. Anesthesiology 86:836-847, 1997

8. Glen JB: Animal studies of the anaesthetic activity of ICI 35 868. Br J Anaesth 52:731-742, 1980

9. Haghighi SS, Green KD, Oro JJ, Drake RK, Kracke GR: Depressive effect of isoflurane anesthesia on motor evoked potentials. Neurosurgery 26:993-997, 1990

10. Haghighi SS, Sirintrapun SJ, Keller BP, Oro JJ, Madsen R: Effect of desflurane anesthesia on transcortical motor evoked potentials. J Neurosurg Anesthesiol 8:47-51, 1996

11. Holland NR: Intraoperative electromyography during thoracolumbar spinal surgery. Spine (Phila Pa 1976) 23:19151922, 1998

12. Jellinek D, Jewkes D, Symon L: Noninvasive intraoperative monitoring of motor evoked potentials under propofol anesthesia: effects of spinal surgery on the amplitude and latency of motor evoked potentials. Neurosurgery 29:551-557, 1991

13. Kakinohana M, Motonaga E, Taira Y, Okuda Y: [The effects of intravenous anesthetics, propofol, fentanyl and ketamine on the excitability of spinal motoneuron in human: an Fwave study.] Masui 49:596-601, 2000 (Jpn) 
14. Kalkman CJ, Drummond JC, Ribberink AA, Patel PM, Sano T, Bickford RG: Effects of propofol, etomidate, midazolam, and fentanyl on motor evoked responses to transcranial electrical or magnetic stimulation in humans. Anesthesiology 76:502-509, 1992

15. Kammer T, Rehberg B, Menne D, Wartenberg HC, Wenningmann I, Urban BW: Propofol and sevoflurane in subanesthetic concentrations act preferentially on the spinal cord: evidence from multimodal electrophysiological assessment. Anesthesiology 97:1416-1425, 2002

16. Kawaguchi M, Inoue S, Kakimoto M, Kitaguchi K, Furuya $\mathrm{H}$, Morimoto T, et al: The effect of sevoflurane on myogenic motor-evoked potentials induced by single and paired transcranial electrical stimulation of the motor cortex during nitrous oxide/ketamine/fentanyl anesthesia. J Neurosurg Anesthesiol 10:131-136, 1998

17. Kearse LA Jr, Rosow C, Zaslavsky A, Connors P, Dershwitz M, Denman W: Bispectral analysis of the electroencephalogram predicts conscious processing of information during propofol sedation and hypnosis. Anesthesiology 88:25-34, 1998

18. Kombos T, Suess O, Ciklatekerlio O, Brock M: Monitoring of intraoperative motor evoked potentials to increase the safety of surgery in and around the motor cortex. J Neurosurg 95:608-614, 2001

19. Krieg SM, Schäffner M, Shiban E, Droese D, Obermüller T, Gempt J, et al: Reliability of intraoperative neurophysiological monitoring using motor evoked potentials during resection of metastases in motor-eloquent brain regions: clinical article. J Neurosurg 118:1269-1278, 2013

20. Loughnan BA, Anderson SK, Hetreed MA, Weston PF, Boyd SG, Hall GM: Effects of halothane on motor evoked potential recorded in the extradural space. Br J Anaesth 63:561-564, 1989

21. Maurette P, Simeon F, Castagnera L, Esposito J, Macouillard G, Heraut LA: Propofol anaesthesia alters somatosensory evoked cortical potentials. Anaesthesia 43 Suppl:44-45, 1988

22. Mikuni N, Okada T, Enatsu R, Miki Y, Hanakawa T, Urayama $S$, et al: Clinical impact of integrated functional neuronavigation and subcortical electrical stimulation to preserve motor function during resection of brain tumors. J Neurosurg 106:593-598, 2007

23. Nathan N, Tabaraud F, Lacroix F, Mouliès D, Viviand X, Lansade A, et al: Influence of propofol concentrations on multipulse transcranial motor evoked potentials. Br J Anaesth 91:493-497, 2003

24. Orser BA, Wang LY, Pennefather PS, MacDonald JF: Propofol modulates activation and desensitization of GABAA receptors in cultured murine hippocampal neurons. J Neurosci 14:7747-7760, 1994

25. Scheufler KM, Zentner J: Total intravenous anesthesia for intraoperative monitoring of the motor pathways: an integral view combining clinical and experimental data. J Neurosurg 96:571-579, 2002

26. Sebel PS, Lang E, Rampil IJ, White PF, Cork R, Jopling M, et al: A multicenter study of bispectral electroencephalogram analysis for monitoring anesthetic effect. Anesth Analg 84:891-899, 1997

27. Sigl JC, Chamoun NG: An introduction to bispectral analysis for the electroencephalogram. J Clin Monit 10:392-404, 1994

28. Sihle-Wissel M, Scholz M, Cunitz G: Transcranial magneticevoked potentials under total intravenous anaesthesia and nitrous oxide. Br J Anaesth 85:465-467, 2000

29. Taniguchi M, Nadstawek J, Langenbach U, Bremer F, Schramm J: Effects of four intravenous anesthetic agents on motor evoked potentials elicited by magnetic transcranial stimulation. Neurosurgery 33:407-415, 1993

\section{Disclosures}

The authors report no conflict of interest concerning the materials or methods used in this study or the findings specified in this paper.

\section{Author Contributions}

Conception and design: Mikuni, Ohtaki. Acquisition of data: Ohtaki, Kanno, Noshiro. Analysis and interpretation of data: Ohtaki. Drafting the article: Ohtaki. Critically revising the article: Akiyama. Reviewed submitted version of manuscript: Mikuni, Akiyama. Statistical analysis: Ohtaki. Administrative/technical/ material support: Hayase, Yamakage.

\section{Correspondence}

Nobuhiro Mikuni, Department of Neurosurgery, Sapporo Medical University, South 1, West 16, Chuo-ku, Sapporo, Hokkaido 0608543, Japan. email: mikunin@sapmed.ac.jp. 